\title{
Optical circular polarization in quasars ${ }^{\star} \star \star \star$
}

\author{
D. Hutsemékers ${ }^{1, \star \star \star}$, B. Borguet ${ }^{2}$, D. Sluse ${ }^{3}$, R. Cabanac ${ }^{4}$, and H. Lamy ${ }^{5}$ \\ 1 Institut d'Astrophysique et de Géophysique, Université de Liège, Allée du 6 Août 17, B5c, 4000 Liège, Belgium \\ e-mail: hutsemekers@astro.ulg.ac.be \\ 2 Department of Physics, Virginia Polytechnic and State University, Blacksburg, VA 24061, USA \\ 3 Zentrum für Astronomie der Universität Heidelberg, Mönchhofstr. 12-14, 69120 Heidelberg, Germany \\ ${ }_{5}^{4}$ Laboratoire d'Astrophysique de Toulouse-Tarbes, Université de Toulouse, 57 avenue d'Azereix, 65000 Tarbes, France \\ 5 Institut Belge d'Aéronomie Spatiale, Avenue Circulaire 3, 1180 Bruxelles, Belgium
}

Received 7 July 2010 / Accepted 19 September 2010

\section{ABSTRACT}

\begin{abstract}
We present new optical circular polarization measurements with typical uncertainties $<0.1 \%$ for a sample of 21 quasars. All but two objects have null circular polarization. We use this result to constrain the polarization due to photon-pseudoscalar mixing along the line of sight. We detect significant $(>3 \sigma)$ circular polarization in two blazars with high linear polarization and discuss the implications of this result for quasar physics. In particular, the recorded polarization degrees may be indicative of magnetic fields as strong as $1 \mathrm{kG}$ or a significant contribution of inverse Compton scattering to the optical continuum.
\end{abstract}

Key words. quasars: general - polarization

\section{Introduction}

To interpret the large-scale alignments of quasar optical polarization vectors observed at redshifts $z \sim 1$ (Hutsemékers 1998; Hutsemékers \& Lamy 2001; Hutsemékers et al. 2005) polarization induced by photon-pseudoscalar mixing along the line of sight has been invoked (Hutsemékers 1998; Jain et al. 2002). Photon-pseudoscalar mixing generates dichroism and birefringence, the latter transforming linear polarization into circular polarization and vice-versa along the line of sight. If photonpseudoscalar mixing produces the linear polarization needed to explain the observed alignments, a comparable amount of circular polarization would be expected (Raffelt \& Stodolsky 1988; Jain et al. 2002; Das et al. 2005; Gnedin et al. 2007; Hutsemékers et al. 2008; Payez et al. 2008). Hence, we present accurate circular polarization measurements for a sample of quasars whose polarization vectors are coherently oriented.

The optical circular polarization of quasars has rarely been measured. Our new observations, data reduction, and a compilation of published measurements are presented in Sect. 2. Implications for the photon-pseudoscalar mixing mechanism are discussed in Sect. 3.1. The detection of significant circular polarization in two objects and its consequence for quasar physics are presented in Sect. 3.2.

\section{Observations and data reduction}

The observations were carried out on April 18-20, 2007 at the European Southern Observatory (ESO, La Silla) using the $3.6 \mathrm{~m}$

* Based on observations made with ESO Telescopes at the La Silla Observatory (Chile). ESO program ID: 79.A-0625(B).

$\star \star$ Appendices are only available in electronic form at http: //www . aanda.org

$\star \star \star$ Maître de Recherches au F.R.S.-FNRS. telescope equipped with the ESO Faint Object Spectrograph and Camera EFOSC2. Circular polarization was measured using a super-achromatic quarter-wave $(\lambda / 4)$ retarder plate $(\mathrm{QWP})$, which transforms the circular polarization into linear polarization, and a Wollaston prism, which splits the linearly polarized beam into two orthogonally polarized images of the object (Saviane et al. 2007). The CCD was used in unbinned mode, which corresponds to a scale of $0.157^{\prime \prime} /$ pixel on the sky. All measurements were performed through a Bessel $V$ filter (V\#641; central wavelength: $5476 \AA$; FWHM: $1132 \AA$ ).

At least one pair of exposures with the QWP rotated to the angles $-45^{\circ}$ and $+45^{\circ}$ was secured for each target. Frames were dark-subtracted and flat-fielded. The circular polarization $p_{\mathrm{o}}$, i.e., the normalized Stokes $V / I$ parameter, was extracted from each pair of frames using a procedure used to measure the normalized Stokes $Q / I$ and $U / I$ parameters and described in Lamy \& Hutsemékers (1999) and Sluse et al. (2005). Errors were estimated from the photon noise. Seeing was typically around 1 ". Owing to the variable atmospheric extinction (thin to thick cirrus), some exposures had to be repeated to reach a sufficient signal-to-noise ratio.

The performances of the instrument were checked during our run and during the setup night (April 17) using an unpolarized standard star and a star with high and slowly variable circular polarization, LP 790-20 (West 1989; Jordan \& Friedrich 2002). The results, discussed in Saviane et al. (2007), demonstrated the quality of the instrumental setup. LP 790-20 was also used to fix the sign of the circular polarization, i.e., $p_{\text {circ }}>0$ when the electric vector rotates counter-clockwise as seen by an observer facing the object.

To evaluate the cross-talk between linear and circular polarization, we measured the circular polarization of linearly polarized stars. These observations were repeated several times during our observing run. Hilt 652 was observed during the setup 
Table 1. The circular polarization of linearly polarized standard stars.

\begin{tabular}{lccr}
\hline \hline Object & $p_{\text {lin }}(\%)$ & $\theta_{\text {lin }}\left({ }^{\circ}\right)$ & $p_{\text {circ }}(\%)$ \\
\hline Hilt 652 & $6.25 \pm 0.03$ & $179.2 \pm 0.2^{a}$ & $0.003 \pm 0.021$ \\
Ve 6-23 & $8.26 \pm 0.05$ & $171.6 \pm 0.2^{a}$ & $-0.050 \pm 0.035$ \\
HD155197 & $4.38 \pm 0.03$ & $103.2 \pm 0.2^{b}$ & $0.033 \pm 0.025$ \\
\hline
\end{tabular}

Notes. All these polarization measurements were obtained in the $V$ filter. References for linear polarization: ${ }^{(a)}$ Fossati et al. (2007); (b) Turnshek et al. (1990).

Table 2. New circular polarization measurements of quasars

\begin{tabular}{lcccr}
\hline \hline Object & $z$ & $p_{\text {lin }}(\%)$ & $\theta_{\operatorname{lin}}\left(^{\circ}\right)$ & $p_{\text {circ }}(\%)$ \\
\hline $1120+019$ & 1.465 & $1.95 \pm 0.27$ & $9 \pm 4^{c}$ & $-0.02 \pm 0.05$ \\
$1124-186$ & 1.048 & $11.68 \pm 0.36$ & $37 \pm 1^{g}$ & $-0.04 \pm 0.08$ \\
$1127-145$ & 1.187 & $1.30 \pm 0.40[\mathrm{w}]$ & $23 \pm 10^{a}$ & $-0.05 \pm 0.05$ \\
$1157+014$ & 1.990 & $0.76 \pm 0.18$ & $39 \pm 7^{f}$ & $-0.10 \pm 0.08$ \\
$1205+146$ & 1.640 & $0.83 \pm 0.18$ & $161 \pm 6^{f}$ & $-0.10 \pm 0.09$ \\
$1212+147$ & 1.621 & $1.45 \pm 0.30$ & $24 \pm 6^{c}$ & $0.15 \pm 0.09$ \\
$1215-002^{\star}$ & 0.420 & $23.94 \pm 0.70$ & $91 \pm 1^{g}$ & $-0.42 \pm 0.40$ \\
$1216-010$ & 0.415 & $11.20 \pm 0.17$ & $100 \pm 1^{g}$ & $-0.01 \pm 0.07$ \\
$1222+228$ & 2.058 & $0.92 \pm 0.14$ & $169 \pm 4^{g}$ & $0.01 \pm 0.10$ \\
$1244-255$ & 0.633 & $8.40 \pm 0.20[\mathrm{w}]$ & $110 \pm 1^{a}$ & $-0.23 \pm 0.20$ \\
$1246-057$ & 2.236 & $1.96 \pm 0.18[\mathrm{w}]$ & $149 \pm 3^{e}$ & $0.01 \pm 0.03$ \\
$1254+047$ & 1.024 & $1.22 \pm 0.15[\mathrm{w}]$ & $165 \pm 3^{b}$ & $-0.02 \pm 0.04$ \\
$1256-229^{\star}$ & 0.481 & $22.32 \pm 0.15$ & $157 \pm 1^{g}$ & $0.18 \pm 0.04$ \\
$1309-056$ & 2.212 & $0.78 \pm 0.28$ & $179 \pm 11^{c}$ & $-0.08 \pm 0.06$ \\
$1331-011$ & 1.867 & $1.88 \pm 0.31$ & $29 \pm 5^{c}$ & $-0.04 \pm 0.06$ \\
$1339-180$ & 2.210 & $0.83 \pm 0.15$ & $20 \pm 5^{g}$ & $-0.01 \pm 0.07$ \\
$1416-129$ & 0.129 & $1.63 \pm 0.15[\mathrm{w}]$ & $44 \pm 3^{b}$ & $0.05 \pm 0.06$ \\
$1429-008$ & 2.084 & $1.00 \pm 0.29$ & $9 \pm 9^{c}$ & $0.02 \pm 0.08$ \\
$2121+050$ & 1.878 & $10.70 \pm 2.90[\mathrm{w}]$ & $68 \pm 6^{a}$ & $0.02 \pm 0.15$ \\
$2128-123$ & 0.501 & $1.90 \pm 0.40[\mathrm{w}]$ & $64 \pm 6^{d}$ & $-0.04 \pm 0.03$ \\
$2155-152$ & 0.672 & $22.60 \pm 1.10[\mathrm{w}]$ & $7 \pm 2^{a}$ & $-0.35 \pm 0.10$ \\
\hline
\end{tabular}

Notes. Linear and circular polarizations were measured in the $V$ filter except a series of linear polarization data from the literature measured in white light and noted [w]; ${ }^{(\star))} 1215-002$ is classified as a BL Lac by Collinge et al. (2005); Sbarufatti et al. (2005) re-determined the redshift of 1256-229 $(z=0.481)$ and considered this object as a BL Lac. References for linear polarization: ${ }^{(a)}$ Impey \& Tapia (1990); ${ }^{(b)}$ Berriman et al. (1990); ${ }^{(c)}$ Hutsemékers et al. (1998); ${ }^{(d)}$ Visvanathan \& Wills (1998); ${ }^{(e)}$ Schmidt \& Hines (1999); ${ }^{(f)}$ Lamy \& Hutsemékers (2000); ${ }^{(g)}$ Sluse et al. (2005).

night. The results are given in Table 1 together with the published linear polarization (i.e. the polarization degree $p_{\text {lin }}$ and the polarization position angle $\left.\theta_{\text {lin }}\right)$. Uncertainties are smaller than in Saviane et al. (2007) because of the availability of repeated observations. Although the objects are highly linearly polarized, we measure a null circular polarization. Combining the data of Hilt 652 and Ve 6-23, which have similar polarization angles, we derive the $3 \sigma$ upper limit to the circular polarization due to cross-talk in the $V$ filter $\left|p_{\text {circ }} / p_{\text {lin }}\right| \lesssim 0.0075$.

Our new measurements of quasar circular polarization are reported in Table 2 with $1 \sigma$ photon-noise errors. The targets are extracted from the sample of 355 polarized quasars defined in Hutsemékers et al. (2005), as well as their B1950 names/coordinates, their redshift $z$, and their linear polarization degree and angle, $p_{\text {lin }}$ and $\theta_{\text {lin }}$.

A compilation of other measurements of quasar optical circular polarization is given in Table 3. Unless indicated otherwise, these measurements were obtained in white light, i.e., in the $3200-8800 \AA$ or $4000-8800 \AA$ spectral ranges, which
Table 3. Previous circular polarization measurements of quasars and BL Lac objects.

\begin{tabular}{lcccr}
\hline \hline Object & $z$ & $p_{\text {lin }}(\%)$ & $\theta_{\text {lin }}\left(^{\circ}\right)$ & $p_{\text {circ }}(\%)$ \\
\hline $0237-233$ & 2.223 & $0.25 \pm 0.29$ & $-^{d}$ & $-0.06 \pm 0.08^{a}$ \\
$0955+326$ & 0.533 & $0.18 \pm 0.24$ & $-^{c}$ & $0.06 \pm 0.08^{a}$ \\
$1127-145$ & 1.187 & $1.30 \pm 0.40$ & $23 \pm 10^{e}$ & $0.32 \pm 0.20^{a}$ \\
$1156+295$ & 0.729 & $2.68 \pm 0.41$ & $114 \pm 4^{f}$ & $0.12 \pm 0.14^{b}$ \\
$1222+228$ & 2.058 & $1.09 \pm 0.16[\mathrm{u}]$ & $167 \pm 4^{i}$ & $0.23 \pm 1.80^{i}$ \\
$1226+023$ & 0.158 & $0.25 \pm 0.04$ & $58 \pm 4^{c}$ & $-0.01 \pm 0.02^{g}$ \\
$1253-055$ & 0.536 & $9.00 \pm 0.40$ & $67 \pm 1^{e}$ & $0.09 \pm 0.07^{a}$ \\
$1308+326$ & 0.997 & $12.10 \pm 1.50$ & $68 \pm 3^{e}$ & $-0.08 \pm 0.17^{b}$ \\
$1634+706$ & 1.334 & $0.24 \pm 0.07[\mathrm{u}]$ & $4 \pm 8^{i}$ & $-0.05 \pm 0.09^{i}$ \\
$1641+399$ & 0.594 & $4.00 \pm 0.30$ & $103 \pm 2^{e}$ & $-0.05 \pm 0.23^{b}$ \\
$2230+114$ & 1.037 & $7.30 \pm 0.30$ & $118 \pm 1^{e}$ & $-0.05 \pm 0.17^{a}$ \\
$2302+029$ & 1.044 & $0.66 \pm 0.12[\mathrm{u}]$ & $136 \pm 5^{i}$ & $-0.39 \pm 0.16^{i}$ \\
$0138-097$ & 0.733 & $3.60 \pm 1.50$ & $168 \pm 11^{e}$ & $0.25 \pm 0.35^{k}$ \\
$0219+428$ & 0.444 & $26.11 \pm 0.19$ & $3 \pm 1^{k}$ & $0.16 \pm 0.05^{k}$ \\
$0422+004$ & 0.310 & $10.29 \pm 0.23$ & $179 \pm 1^{g}$ & $0.14 \pm 0.07^{g}$ \\
$0735+178$ & 0.424 & $11.69 \pm 0.22$ & $123 \pm 1^{k}$ & $0.03 \pm 0.05^{k}$ \\
$0823-223$ & 0.910 & $14.39 \pm 0.16$ & $11 \pm 1^{k}$ & $0.16 \pm 0.08^{k}$ \\
$0851+202$ & 0.306 & $10.80 \pm 0.30$ & $156 \pm 1^{e}$ & $-0.01 \pm 0.02^{a}$ \\
$1101+384$ & 0.031 & $2.59 \pm 0.11$ & $10 \pm 1^{h}$ & $0.02 \pm 0.03^{h}$ \\
$2155-304$ & 0.116 & $4.12 \pm 0.25$ & $93 \pm 2^{j}$ & $-0.02 \pm 0.02^{j}$ \\
$2200+420$ & 0.068 & $4.90 \pm 0.40$ & $147 \pm 2^{e}$ & $-0.07 \pm 0.19^{a}$ \\
\hline
\end{tabular}

Notes. All but a few polarization measurements were obtained in white light; the multi-color measurements in references $(h),(j)$ and $(k)$ were averaged; $[\mathrm{u}]$ refers to linear and circular polarization measurements averaged over the 2200-3200 $\AA$ ultraviolet wavelength band; the circular polarization of objects $2200+420$ and $2230+114$ was obtained in the 4000-6000 $\AA$ and 3500-5200 $\AA$ bands respectively; italicized names indicate objects classified as BL Lac in Véron-Cetty \& Véron (2006). References for linear and circular polarization: ${ }^{(a)}$ Landstreet \& Angel (1972); ${ }^{(b)}$ Moore \& Stockman (1981); ${ }^{(c)}$ Stockman et al. (1984); ${ }^{(d)}$ Moore \& Stockman (1984); ${ }^{(e)}$ Impey \& Tapia (1990); ${ }^{(f)}$ Wills et al. (1992); ${ }^{(g)}$ Valtaoja et al. (1993); ${ }^{(h)}$ Takalo \& Sillanpää (1993); ${ }^{(i)}$ Impey et al. (1995); ${ }^{(j)}$ Tommasi et al. (2001a); ${ }^{(k)}$ Tommasi et al. (2001b).

roughly correspond to an effective wavelength of $6000 \AA$. When several estimates of either linear or circular polarization are available, only the value with the smallest uncertainty is considered. BL Lac objects, similar in many respects to highly polarized quasars (HPQs) (e.g., Scarpa \& Falomo 1997; Fan et al. 2008), are included. Both BL Lac and HPQs belong to the blazar sub-group of active galactic nuclei (AGN). For BL Lac objects, the polarization is often strongly variable. We then adopt the circular polarization with the smallest uncertainty and, when quasisimultaneous observations are available, the value of the linear polarization obtained as close as possible in time. Otherwise we select a representative value of the linear polarization from the survey of Impey \& Tapia (1990).

\section{Discussion}

The measurements reported in Tables 2 and 3 show that all quasars and BL Lac objects have null circular polarization $(<3 \sigma)$ except two HPQs, 1256-229 and 2155-152, and one highly polarized BL Lac object, 0219+428.

We first discuss the constraints provided by the majority of null detections on the photon-pseudoscalar mixing mechanism, and then the consequences of the three detections for blazar physics. 


\subsection{Constraints on photon-pseudoscalar mixing}

Quasars with right ascension between $11^{\mathrm{h}} 20^{\mathrm{m}}$ and $14^{\mathrm{h}} 30^{\mathrm{m}}$ belong to the region of alignment A1 defined in Hutsemékers (1998). In this region of the sky, quasars with $1<z<2.3$ have their polarization angle preferentially in the range $\left[146^{\circ}-226^{\circ}\right]$ (modulo $180^{\circ}$ ), while quasars with $0<z<1$ have their polarization angle preferentially in the range $\left[30^{\circ}-120^{\circ}\right]$. Assuming that the quasar intrinsic polarization vectors are randomly oriented, the addition of a small systematic linear polarization $\Delta p_{\text {lin }} \simeq 0.5 \%$ at a fixed position angle can account for the observed alignments (Hutsemékers et al. 2008, Appendix A). If photon-pseudoscalar mixing is responsible for this extra linear polarization, one expects, on average, that $\left|p_{\text {circ }}\right| \simeq \Delta p_{\text {lin }} \simeq 0.5 \%$ (Appendix B). Because the light from most quasars is intrinsically linearly polarized to some extent and not circularly polarized, limits on any additional polarization from interactions along the line of sight cannot be derived from the measurement of the linear polarization degree, while, on the other hand, useful constraints can be derived from the measurement of circular polarization.

Most of the thirteen quasars with $z>1$ located in region A1 were found to have $\left|p_{\text {circ }}\right| \lesssim 0.25 \%$ ( $3 \sigma$ upper limit), which is definitely smaller than the expected value. Averaging over the thirteen objects, we infer that $\left\langle\left|p_{\text {circ }}\right|\right\rangle=0.035 \pm 0.016 \%$ after neglecting the sign, from which a stringent $3 \sigma$ upper limit on the circular polarization of $\left\langle\left|p_{\text {circ }}\right|\right\rangle \leq 0.05 \%$ can be derived. This limit is one order of magnitude smaller than the expected value $\left|p_{\text {circ }}\right| \simeq 0.5 \%$. A similar result is obtained for the nine objects at $z<1$ in that region.

This result rules out the interpretation of the observed alignments in terms of photon-pseudoscalar mixing, at least in its simplest formulation. A more complex treatment of the photonpseudoscalar interaction is thus required to account for the observations (Payez et al. 2010a,b).

\subsection{Detection of optical circular polarization and implication for blazar physics}

Circular polarization is detected at the $3 \sigma$ level in two HPQs: 1256-229 and 2155-152 (Table 2). On April 21, we had the opportunity to re-measure the linear polarization of these objects in the $V$ filter, after replacing the quarter-wave plate by the half-wave plate (HWP) (cf. Saviane et al. 2007). Four exposures with the HWP rotated to $0^{\circ}, 22.5^{\circ}, 45^{\circ}$, and $67.5^{\circ}$ were secured and reduced in the standard way (e.g. Sluse et al. 2005). The results are reported in Table 4, together with the circular polarization measurements from Table 2. Although the optical linear polarization of these quasars is high, the circular polarization we measured at the same epoch is above the $3 \sigma$ upper limit on the circular polarization generated by the instrumental cross-talk (Sect. 2).

In Table 4, we also summarize the main polarization properties of these objects, including measurements at radio wavelengths. For completeness, we include the BL Lac object for which circular polarization was found to be significant after averaging the UBVRI measurements (Table 3). As far as we know, these are the only $3 \sigma$ detections of optical circular polarization in quasars, in addition to those reported by Wagner \& Mannheim (2001) for $3 \mathrm{C}_{279^{1}}(=1253-055)$. Although variable (as commonly seen in HPQs), the optical linear polarization is high in

\footnotetext{
1 Wagner et al. (2000) reported that $p_{\text {circ }}=0.25 \pm 0.03 \%$, while Wagner $\&$ Mannheim (2001) reported $p_{\text {circ }}=0.45 \pm 0.03 \%$. These detections
}

Table 4. Radio to optical polarization characteristics of objects with detected optical circular polarization.

\begin{tabular}{|c|c|c|c|c|}
\hline \multicolumn{4}{|c|}{$1256-229(\mathrm{PKS}) z=0.481$} & \multirow{2}{*}{$\frac{\text { HPQ, BL Lac? }}{p_{\text {circ }}(\%)}$} \\
\hline Date & $v(\mathrm{Ghz})$ & $p_{\text {lin }}(\%)$ & $\theta_{\operatorname{lin}}\left({ }^{\circ}\right)$ & \\
\hline $03 / 2002$ & $5.4 \times 10^{5}$ & $22.32 \pm 0.15$ & $157 \pm 1^{d}$ & - \\
\hline $04 / 2007$ & $5.4 \times 10^{5}$ & 15. & 163 & $0.18 \pm 0.04^{j}$ \\
\hline \multicolumn{4}{|c|}{$2155-152(\mathrm{PKS}) z=0.672$} & $\overline{\mathrm{HPQ}}$ \\
\hline Date & $v(\mathrm{Ghz})$ & $p_{\text {lin }}(\%)$ & $\theta_{\operatorname{lin}}\left({ }^{\circ}\right)$ & $p_{\text {circ }}(\%)$ \\
\hline $08 / 1984$ & $5.4 \times 10^{5}$ & $32.70 \pm 1.30$ & $6 \pm 1^{a}$ & - \\
\hline $04 / 2$ & $5.4 \times 10^{5}$ & 17. & $51 \pm 1^{j}$ & $-0.35 \pm 0.10^{j}$ \\
\hline 09/2007 & 85.6 & 11.0 & $52 \pm 1^{i}$ & $<0.93^{i}$ \\
\hline $03 / 2003$ & 15.4 & 3.66 & $-{ }^{f}$ & $<0.34^{f}$ \\
\hline \multirow[t]{2}{*}{ 1979-1999 } & 8.0 & 4.81 (mean) & $--^{h}$ & - \\
\hline & & $15.3(\max )$ & $-^{h}$ & - \\
\hline \multicolumn{4}{|c|}{$0219+428(3 \mathrm{C} 66 \mathrm{~A}) z=0.444$} & BL Lac \\
\hline Date & $v(\mathrm{Ghz})$ & $p_{\text {lin }}(\%)$ & $\theta_{\operatorname{lin}}\left({ }^{\circ}\right)$ & $p_{\text {circ }}(\%)$ \\
\hline $01 / 19$ & $5 \times 10^{5}[\mathrm{w}]$ & $31.07 \pm 0.3$ & $39 \pm 1^{b}$ & $0.76 \pm 0.10^{b}$ \\
\hline $12 / 1999$ & $5 \times 10^{5}[\mathrm{w}]$ & 26.1 & & $0.16 \pm 0.05^{c}$ \\
\hline $11 / 2008$ & 85.6 & $4.36 \pm 0$ & $3 \pm 4^{i}$ & $<0.73^{i}$ \\
\hline $12 / 199$ & 5. & 0 & $-^{e}$ & $<0.20^{e}$ \\
\hline 1974-1999 & 8.0 & 2.96 (mean) & $-g$ & - \\
\hline
\end{tabular}

Notes. Multi-color measurements in references $(b)$ and $(c)$ were averaged; upper limits are given at $3 \sigma$. References for linear or circular polarization: ${ }^{(a)}$ Brindle et al. (1986); ${ }^{(b)}$ Takalo \& Sillanpää (1993); ${ }^{(c)}$ Tommasi et al. (2001b); ${ }^{(d)}$ Sluse et al. (2005); ${ }^{(e)}$ Homan et al. (2001); (f) Homan \& Lister (2006); ${ }^{(g)}$ Fan et al. (2006); ${ }^{(h)}$ Fan et al. (2008); (i) Agudo et al. (2010); ${ }^{(j)}$ this work.

all three objects suggesting that a relation exists between linear and circular polarization.

Radio circular polarization has been detected in a small number of blazars with typical values of a few tenths of a percent (Weiler \& de Pater 1983; Rayner et al. 2000; Homan et al. 2001; Homan \& Lister 2006; Vistrishchak et al. 2008). Although the origin of the radio circular polarization is not yet understood, two main mechanisms of production have been proposed: intrinsic circular polarization of the relativistically beamed synchrotron radiation (which also produces the radio linear polarization) and Faraday conversion of linear to circular polarization (e.g. Wardle \& Homan 2003). Since beamed synchrotron radiation can also explain the high optical linear polarization observed in HPQs and contribute significantly to the optical continuum (Impey \& Tapia 1990; Wills et al. 1992), a similar origin to both the optical and the radio circular polarizations appears likely although they most probably arise from different regions. Since Faraday conversion is inefficient at visible wavelengths, the detected optical circular polarization should be caused by synchrotron emission. Since intrinsic circular polarization is not produced in a positron-electron plasma, this mechanism requires the predominance of a proton-electron plasma, as already suggested by circular polarization measurements obtained at millimeter wavelengths (Agudo et al. 2010). Furthermore, if circular polarization is intrinsic, a correlation between the linear and the circular polarization degrees is expected, the high recorded values indicating a rather homogeneous magnetic field whose strength should be of the order of $1 \mathrm{kG}$ (e.g. Valtaoja et al. 1993). This is much higher than usually assumed in quasar jets, and can only occur in small regions close to the quasar core (Wardle \& Homan 2003; Silant'ev et al. 2009; Piotrovich et al. 2010). On

were considered tentative by the authors in view of significant instrumental effects and thus not included in Tables 3 and 4. 
the other hand, the optical continuum could predominantly arise from inverse Compton scattering of radio synchrotron radiation, a mechanism that preserves the circular polarization (Sciama $\&$ Rees 1967). This would require a significant circular polarization at radio wavelengths, which is apparently not observed (Table 4). Given the uncertainties and the non-simultaneous observations, no firm conclusion can be derived. Unveiling the origin of the optical circular polarization - even a few tenths of a percent - thus appears challenging (see also Rieger \& Mannheim 2005). A clearer understanding would require simultaneous observations at radio and optical wavelengths.

\section{Conclusions}

We have reported new accurate measurements of optical circular polarization in the $V$ filter for a sample of 21 quasars. For most objects, the uncertainties are smaller than $0.1 \%$, and smaller than $0.05 \%$ for six of them.

All objects have null polarization within the uncertainties except two highly linearly polarized blazars. This has allowed us to constrain the polarization caused by photon-pseudoscalar mixing along the line of sight, ruling out the interpretation of the observed alignments of quasar polarization vectors in terms of photon-pseudoscalar mixing, at least in the framework of a simple formulation.

We also found small but significant optical circular polarization in two blazars, providing clues about the strength of the magnetic fields, the nature of the jets and/or the dominant emission mechanism. Our observations demonstrate that optical circular polarization is routinely measurable with present day highaccuracy polarimeters.

Acknowledgements. D.H. thanks Alexandre Payez and Jean-René Cudell for useful discussions. A fellowship from the Alexander von Humboldt Foundation to D.S. is gratefully acknowledged. This research has made use of data originally from the University of Michigan Radio Astronomy Observatory, which has been supported by the University of Michigan and the National Science Foundation.

\section{References}

Agudo, I., Thum, C., Wiesemeyer, H., \& Krichbaum, T. P. 2010, ApJS, 189, 1 Berriman, G., Schmidt, G. D., West, S. C., \& Stockman, H. S. 1990, ApJS, 74, 869

Brindle, C., Hough, J. H., Bailey, J. A., Axon, D. J., \& Hyland, A. R. 1986, MNRAS, 221, 739

Collinge, M. J., Strauss, M. A., Hall, P. B., et al. 2005, AJ, 129, 2542

Das, S., Jain, P., Ralston, J. P., \& Saha, R. 2005, JCAP, 06, 002

Fan, J.-H., Hua, T.-X., Yuan, Y.-H., et al. 2006, PASJ, 58, 94

Fan, J.-H., Yuan, Y.-H., Hua, T.-X., et al. 2008, PASJ, 60, 707
Gnedin, Y. N., Piotrovich, M. Y., \& Natsvlishvili, T. M. 2007, MNRAS, 374, 276

Fossati, L., Bagnulo, S., Mason, E., \& Landi Degl'Innocenti, E. 2007, ASPC 364,503

Homan, D. C., Attridge, J. M., \& Wardle, J. F. C. 2001, ApJ, 556, 113

Homan, D. C., \& Lister, M. L. 2006, AJ, 131, 1262

Hutsemékers, D. 1998, A\&A, 332, 410

Hutsemékers, D., \& Lamy, H. 2001, A\&A, 367, 381

Hutsemékers, D., Lamy, H., \& Remy, M. 1998, A\&A, 340, 371

Hutsemékers, D., Cabanac, R., Lamy, H., \& Sluse, D. 2005, A\&A, 441, 915

Hutsemékers, D., Payez, A., Cabanac, R., et al. 2008, in Astronomical

Polarimetry 2008: Science from Small to Large Telescopes, ed. P. Bastien and N. Manset, ASP Conf. Ser., in press [arXiv:0809. 3088]

Impey, C. D., \& Tapia, S. 1990, ApJ, 354, 124

Impey, C. D., Malkan, M. A., Webb, W., \& Petry, C. E. 1995, ApJ, 440, 80

Jain, P., Panda, S., \& Sarala, S. 2002, Phys. Rev. D., 66, 085007

Jordan, S., \& Friedrich, S. 2002, A\&A, 383, 519

Lamy, H., \& Hutsemékers, D. 1999, The Messenger, 96, 25

Lamy, H., \& Hutsemékers, D. 2000, A\&AS, 142, 451

Landstreet, J. D., \& Angel, J. R. P. 1972, ApJ174, L127

Moore, R. L., \& Stockman, H. S. 1981, ApJ, 243, 60

Moore, R. L., \& Stockman, H. S. 1984, ApJ, 279, 465

Payez, A., Cudell, J. R., \& Hutsemékers, D. 2008, AIP Conf. Proc., 1038, 211

Payez, A., Cudell, J. R., \& Hutsemékers, D. 2010a, Proc. of the 5th Patras

Workshop on Axions, WIMPs and WISPs, DESY-PROC-2009-5, 133

Payez, A., Cudell, J. R., \& Hutsemékers, D. 2010b, AIP Conf. Proc., 1241, 444

Piotrovich, M. Y., Gnedin, Y. N., Natsvlishvili, T. M., \& Silant'ev, N. A. 2010, Astron. Lett., 36, 389

Raffelt, G., \& Stodolsky, L. 1988, Phys. Rev. D, 37, 1237

Rayner, D. P., Norris, R. P., \& Sault, R. J. 2000, MNRAS, 319, 484

Rieger, F. K., \& Mannheim, K. 2005, Chin. J. Astron. Astrophys., 5, 311

Saviane, I., Piirola, V., Bagnulo, S., et al. 2007, The Messenger, 129, 14

Sbarufatti, B., Treves, A., Falomo, R., et al. 2005, AJ, 129, 559

Scarpa, R., \& Falomo, R. 1997, A\&A, 325, 109

Sciama, D. W., \& Rees, M. J. 1967, Nature, 216, 147

Schmidt, G. D., \& Hines, D. C. 1999, ApJ, 512, 125

Silant'ev, N. A., Piotrovich, M. Y., Gnedin, Y. N., \& Natsvlishvili, T. M. 2009, A\&A, 507, 171

Sluse, D., Hutsemékers, D., Lamy, H., Cabanac, R., \& Quintana, H. 2005, A\&A, 433, 757

Stockman, H. S., Moore, R. L., \& Angel, J. R. P. 1984, ApJ, 279, 485

Takalo, L. O., \& Sillanpää, A. 1993, Ap\&SS, 206, 191

Tommasi, L., Diaz, R., Pallazi, E., et al. 2001a, ApJS, 132, 73

Tommasi, L., Palazzi, E., Pian, E., et al. 2001b, A\&A, 376, 51

Turnshek, D. A., Bohlin, R. C., Williamson, R. L., et al. 1990, AJ, 99, 1243

Valtaoja, L., Karttunen, H., Valtaoja, E., Shakhovskoy, N. M., \& Efimov, Y. S. 1993, A\&A, 273, 393

Véron-Cetty, M.-P., \& Véron, P. 2006, A\&A, 455, 773

Vitrishchak, V. M., Gabuzda, D. C., Algaba, J. C., et al. 2008, MNRAS, 391, 124

Visvanathan, N., \& Wills, B. J. 1998, AJ, 116, 2119

Wagner, S. J., \& Mannheim, K. 2001, ASPC, 250, 142

Wagner, S. J., Seifert, W., Appenzeller, I., et al. 2000, Proc. SPIE, 4005, 95

Wardle, J. F. C., \& Homan, D.C. 2003, Ap\&SS, 288, 143

Weiler, K. W., \& de Pater, I. 1983, ApJS, 52, 293

West, S. C. 1989, ApJ, 345, 511

Wills, B. J., Wills, D., Breger, M., Antonucci, R. R. J., \& Barvainis, R. 1992, ApJ, 398, 454 

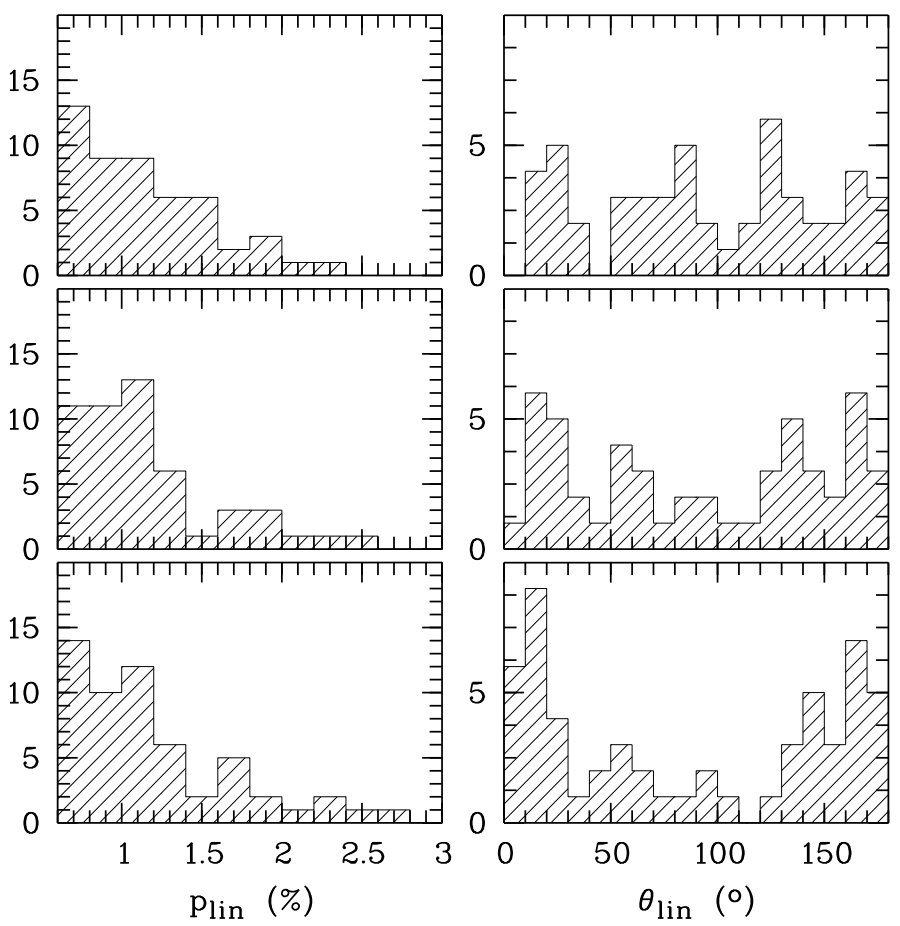

Fig. A.1. The effect of the addition of a small systematic polarization $\Delta p_{\text {lin }}$ on the distributions of the polarization degree $p_{\text {lin }}$ and of the polarization angle $\theta_{\text {lin }}$. From top to bottom, $\Delta p_{\text {lin }}=0 \%, 0.25 \%$, and $0.5 \%$ (see text for details).

\section{Appendix A: The determination of $\Delta p_{\text {lin }}$}

Although partly discussed in previous papers (e.g. Hutsemékers et al. 2005), we provide some details of the simulations performed to estimate $\Delta p_{\text {lin }}$, the small additional linear polarization needed to reproduce the observed alignments of quasar polarization vectors. These simulations extend those discussed in Hutsemékers \& Lamy (2001), accounting for the measurement errors.

We first modeled the distribution of the debiased polarization degree in the full quasar sample (from Hutsemékers et al. 2005, including objects with $p<0.6 \%$ ). We found that it was reasonably well reproduced by a half-gaussian distribution of zero mean and unitary variance. According to this distribution, we randomly generate 80 values of the polarization degree $p$, most of them lying between $0 \%$ and $3 \%$. We also generate 80 values of the polarization angle $\theta$, uniformly distributed between $0^{\circ}$ and $180^{\circ}$. From $p$ and $\theta$, we compute the normalized Stokes parameters $q$ and $u$ to which we add a systematic polarization $\Delta q>0$ (we assume for simplicity that $\Delta u=0$, which corresponds to add linear polarization at $\theta=0^{\circ}$ ). We also add random noise uniformly generated between $-0.2 \%$ and $+0.2 \%$, in agreement with the uncertainties in the measurements (Hutsemékers et al. 2005). Finally, from the modified $q$ and $u$ we recompute $p, \theta$, and $\sigma_{\theta}$ in the usual way and select the good quality measurements with the criteria previously used, i.e., $p \geq 0.6 \%$ and $\sigma_{\theta} \leq 14^{\circ}$. This leaves us with $\sim 60$ polarization values. This is comparable to the number of objects in the alignment region (cf. Fig. 7 of Hutsemékers et al. 2005, to which these simulations should be compared).

The results are illustrated in Fig. A. 1 for $\Delta q=0 \%, 0.25 \%$, and $0.5 \%$ from top to bottom. We see that, in the distribution of polarization angles, a significant deviation to uniformity is only obtained for $\Delta p_{\text {lin }}=\Delta q=0.5 \%$. At the same time, the distribution of the polarization degree does not appear significantly modified. Since this additional polarization at a single polarization angle is likely unrealistic, $\Delta p_{\text {lin }}$ should be seen as a lower limit, although it cannot be much higher. Indeed, much larger values would make the distribution of the polarization degree incompatible with the observations (e.g. Hutsemékers \& Lamy 2001).

\section{Appendix B: Circular polarization due to photon-pseudoscalar mixing}

In the weak mixing case, photons with polarizations parallel to an external magnetic field $\boldsymbol{B}$ that propagate through the distance $L$ can decay into pseudoscalars with a probability

$P_{\gamma a} \simeq(g B l)^{2} \sin ^{2}(\xi / 2)$,

where $l=2 \omega /\left(\omega_{p}^{2}-m_{a}^{2}\right)$ and $\xi=L / l, \omega$ is the photon frequency, $\omega_{p}$ the plasma frequency, $m_{a}$ the pseudoscalar mass, and $g$ the photon-pseudoscalar coupling constant (Raffelt \& Stodolky 1988; Jain et al. 2002). As long as $P_{\gamma a}$ is small, the linear polarization perpendicular to $\boldsymbol{B}$ generated by dichroism can be approximated by $\Delta p_{\text {lin }}=P_{\gamma a}$. The mixing also induces a polarization-dependent phase shift (retardance)

$\phi_{a} \simeq\left(\frac{g B l}{2}\right)^{2}(\xi-\sin \xi)$

acquired by the photons during propagation, which results in circular polarization. As noted by Raffelt \& Stodolky (1988), both effects are on the order of $(g B l)^{2}$.

Assuming $m_{a} \ll \omega_{p}$, we have $l \simeq 4 \times 10^{-14} v n_{\mathrm{e}}^{-1} \mathrm{Mpc}$, where $v$ is the frequency in $\mathrm{GHz}$ and $n_{\mathrm{e}}$ the electronic density in $\mathrm{cm}^{-3}$. At optical wavelengths $\left(v=5 \times 10^{5} \mathrm{GHz}\right)$ and under various conditions (e.g. $n_{\mathrm{e}} \sim 10^{-6} \mathrm{~cm}^{-3}$ and $L \sim 10 \mathrm{Mpc}$ in superclusters, or $n_{\mathrm{e}} \sim 10^{-8} \mathrm{~cm}^{-3}$ and $L \sim 1 \mathrm{Gpc}$ in the intergalactic medium), $\xi=L / l \sim 500$. With a frequency bandwidth $\Delta v / v \sim 0.2$ and $\xi \gg 1$, we find that $\phi_{a} \simeq\left\langle\Delta p_{\text {lin }}\right\rangle \xi / 2 \sim 10^{2}\left\langle\Delta p_{\text {lin }}\right\rangle$, where $\left\langle\Delta p_{\text {lin }}\right\rangle$ represents the average value of $\Delta p_{\text {lin. }}$. Similar estimates are derived when accounting for density fluctuations (Jain et al. 2002).

Adopting the convention of $u=0$ and $q>0$ for polarization vectors parallel to $\boldsymbol{B}$, the dichroism and birefringence induced by photon-pseudoscalar mixing modify the polarization according to

$$
\begin{aligned}
q & =q_{0}-\Delta p_{\text {lin }}, \\
u & =u_{0} \cos \phi_{a}, \\
v & =u_{0} \sin \phi_{a},
\end{aligned}
$$

where $q_{0}$ and $u_{0}$ are the normalized Stokes parameters representing the initial linear polarization state and $v_{0}=0$. Assuming that the sources are initially polarized at $p_{0} \simeq 2 \%$ with randomly oriented polarization angles and that $\left\langle\Delta p_{\text {lin }}\right\rangle<0.01$ (Appendix A), we finally obtain the average circular polarization expected to result from the photon-pseudoscalar mixing: $\langle|v|\rangle \sim(2 / \pi) p_{0} \phi_{a}$, i.e., $\left\langle\left|p_{\text {circ }}\right|\right\rangle \sim\left\langle\Delta p_{\text {lin }}\right\rangle \sim 0.5 \%$. This estimate applies to a variety of plausible situations, in agreement with the simulations shown in Das et al. (2005), Hutsemékers et al. (2008), and Payez et al. (2008). 